\title{
Le cas des beurres végétaux et des cires d'origine naturelle
}

Véronique ROSSOW

Ets B.Rossow et Cie,

92 Avenue du Général de Gaulle,

92635 Gennevilliers CEDEX

<veronique.rossow@rossow.fr>

\begin{abstract}
Natural waxes such as Beeswax, Carnauba wax and Candelilla wax are widely used in the Cosmetic and Food industries. Thanks to their composition, and besides a compulsory high safety, they provide desirable properties such as strength, gloss, emolliency, barrier effect, making them essential in numerous applications. For example, lots of candies are coated with either one or a mix of these 3 waxes, while for Cosmetics, the waxy part of lipsticks contains various combinations of such waxes. Within the cosmetic industry, the "green wave" - natural products - which is developing to the detriment of synthetic or mineral raw materials, has generated know-hows to enlighten new values to natural resources. Several types of developments have occurred: managing to substitute materials facing bad press (ex: petrolatum, lanolin), mimicking trendy "butter-like" textures either by adequate mixture or by chemical reaction to offer alternatives to overused Shea or Cupuaçu Butter, or as a result of more recent research programs, identifying natural waxes acting more like active ingredients than "just" like excipients (Mimosa Flower wax, Lemon Peel or Orange Peel waxes, etc.).
\end{abstract}

Key words: butters, flower waxes, fruit waxes, substitute of petrolatum, substitute of lanoline, massage candle

chimiques, soit par une valorisation de leurs utilisations.

\section{Les nouvelles tendances en cosmétique}

La plupart des fabricants de produits cosmétiques développent aujourd'hui des gammes complètes de produits cosmétiques naturels, qu'ils font pour la plupart certifier par des organismes agréés (Ecocert, Qualité-France, etc.) $[1,2]$. Cette demande croissante a amené les fabricants de matières premières à ajuster leurs axes de recherche et à apporter des produits qui correspondent à ces nouveaux besoins tant par leur origine (naturelle ou végétale) que par leurs procédés de fabrication ("chimie verte »). Depuis ces dernières années, grâce à une disponibilité croissante de matières premières d'origine végétale, l'enjeu du formulateur n'est plus de pouvoir développer une formule cosmétique totalement " verte ", mais de développer une formule verte ayant des propriétés cosmétiques aussi attractives que celles des formulations plus traditionnelles.

La revendication "substance d'origine naturelle ou végétale » s'accompagne donc de plus en plus d'autres revendications plus ciblées qui réfèrent par exemple à l'origine physique de la source (telle que "matière première biologique "), ou bien à son origine géographique (connotation d'exotisme, référence à des coutumes locales, etc.).

Ainsi, dans le cas des beurres et des cires, la plupart des nouveautés correspondent à ces tendances, soit par une recherche de nouvelles sources, ou procédés de fabrication moins

\section{Les beurres}

Par définition, les beurres sont des corps gras solides à température ambiante, qui fondent à des températures proches de celle de la peau. On distinguera les beurres natifs (généralement extraits de noyaux ou de graines, et dont les constituants principaux sont des triglycérides) des beurres "fabriqués", obtenus par divers procédés physiques ou chimiques, et dont les textures sont proches de celles des beurres natifs.

Ces matières premières connaissent un regain d'intérêt auprès des formulateurs de produits cosmétiques naturels, car ils sont faciles à formuler, sont de très bons émollients et participent grandement à l'amélioration du toucher des formules [3]. Les plus connus (beurres de Karité, de mangue, du cupuaçu) rentrent dans la composition aussi bien de produits de soin que de maquillage, auxquels ils apportent onctuosité, glissant, émollience, et permettent d'augmenter l'homogénéité des phases grasses $[4,5]$. L'impact marketing qu'ils apportent à une formulation reste un critère important dans le choix que fera un formulateur d'utiliser une source ou une autre.

Avec la connotation « exotique », on voit apparaître de plus en plus de matières premières d'origines lointaines. Si l'Amérique du Sud, avec notamment le Brésil et l'Amazonie, est toujours très prisée, I'Inde - dont la biodiversité est également très riche - devient source de nouvelles substances très intéressantes.

Ainsi on trouve aujourd'hui proposés aux formulateurs ces trois nouveaux beurres natifs, dont les principales caractéristiques sont données dans le tableau 1.

La composition chimique de ces beurres influe sur leurs propriétés d'usage :

- Le beurre de kokum est un bon candidat pour remplacer le beurre de cacao car il a une composition en triglycérides uniforme [6].

- Le beurre de mowrah, utilisé par les populations locales aussi bien en cosmétique qu'en alimentaire, présente une teneur en AGPI qui en fait un bon candidat pour les formulations « anti-rides » [7].

- Le beurre de sal, par sa fraction d'insaponifiable, est également recommandé dans des formulations hydratantes et anti-âges [8].

Les industriels restent cependant confrontés au besoin de renouveau constant, dans une industrie où le temps de développement d'une nouvelle source d'approvisionnement et le respect des réglementations imposent de trouver d'autres voies que le $100 \%$ naturel.

On voit ainsi de plus en plus de beurres fabriqués être proposés aux formulateurs.

L'objectif étant de créer un produit ayant une texture similaire à celle d'un beurre afin d'apporter de l'onctuosité et de l'émollience, on peut recenser plusieurs procédés physiques et/ou chimiques permettant d'y parvenir :

- Le plus couramment utilisé car le plus simple, consiste à mélanger une huile à des corps gras 
Tableau 1. Compositions et propriétés physico-chimiques de 3 beurres natifs (origine : Inde). D'après [6-8]).

\begin{tabular}{|c|c|c|c|c|}
\hline & & Beurre de kokum & Beurre de mowrah & Beurre de sal \\
\hline Nom INCI & & Garcinia Indica Seed Butter & $\begin{array}{l}\text { Madhuca Latifolia \& } \\
\text { Madhuca Longifolia }\end{array}$ & Shorea Robusta \\
\hline \multirow[t]{4}{*}{ Composition } & AGS & $52-68 \%$ & $36-59 \%$ & $48-63$ \\
\hline & AGMI & $30-42 \%$ & $28-44$ & $37-43$ \\
\hline & AGPI & $0-2 \%$ & $11-20$ & $0-4$ \\
\hline & Insaponifiable & $0,2-1 \%$ & ND & $0.6-2.2$ \\
\hline \multicolumn{5}{|c|}{ Propriétés physico-chimique } \\
\hline & $\begin{array}{l}\text { Point de fusion } \\
\text { (AOCS CC3-25) }\end{array}$ & $37-40^{\circ} \mathrm{C}$ & $34-38^{\circ} \mathrm{C}$ & $34-38^{\circ} \mathrm{C}$ \\
\hline & Indice d'acide (AOCS Cd3a-63) & $<2$ & ND & ND \\
\hline & Indice d'iode (AOCS Tg2a-64) & $30-50$ & $53-70$ & $35-43$ \\
\hline & Indice de saponification (ISO 3657-1988) & $187-193$ & $187-200$ & 178-192 \\
\hline
\end{tabular}

de divers points de fusion, afin d'obtenir le beurre équivalent.

- L'hydrogénation partielle d'une huile permet également d'obtenir une texture proche d'un beurre. Ce procédé industriel présente l'intérêt d'être accepté par des référentiels de certification « produits cosmétiques naturels » tel que Ecocert.

- Le mélange d'une huile et de cette même huile hydrogénée est aussi une autre voie d'obtention.

Mais selon le procédé de fabrication, on pourra sous une même dénomination générique trouver de très grandes différences physicochimiques.

Si I'on prend l'exemple du beurre d'avocat et de son point de fusion : obtenu par mélange $d^{\prime}$ 'huile vierge d'avocat à divers corps gras, son point de fusion de 48,9 à $60^{\circ} \mathrm{C}$ (méthode ASTM D-127) [9], alors qu'il est de 38 à $48^{\circ} \mathrm{C}$ (méthode NFT60-121) quand il est obtenu par hydrogénation partielle d'une huile d'avocat raffinée [10]. Les caractéristiques données ici dépendent également du producteur autant que de la méthode de fabrication utilisée.

Cependant, certaines huiles véhiculent une très bonne image marketing, qui se répercute sur les beurres correspondants (exemple du beurre de germe de blé, beurre de neem, beurre $d^{\prime}$ olive, beurre d'orange, etc.).

\section{Les cires}

Et I'on retrouve cette même démarche pour les cires, puisqu'aujourd'hui, sont proposées sous la terminologie de « cire », des substances natives ou bien fabriquées (par hydrogénation). Les cires sont par définition des composés ayant un point de fusion élevé, et leur composition chimique varie selon les espèces végétales.

Aujourd'hui, les nouvelles cires d'origine naturelle proposées aux formulateurs sont extraites principalement des fruits et des fleurs. Elles jouent alors plus un rôle d'actif que d'excipient par les revendications auxquelles elles sont associées.

- La cire de mimosa (nom INCl : Acacia Decurrens Flower Wax) est de couleur jaune, a un point de fusion de $60-65^{\circ} \mathrm{C}$, un indice d'acide entre 8 et 18 , et un indice de saponification entre 50 et 70 . Elle est principalement constituée d'esters à longues chaînes, et contient des phytostérols [11].

- La cire d'orange (nom $\mathrm{INCl}$ : Orange peel wax) est quant à elle semi-solide, et contient des molécules biologiquement actives telles que des caroténoïdes (pigments naturels, antioxydants et précurseurs de vitamines), des stérols (anti-inflammatoires) et des flavonoïdes (antioxydants, antimicrobiens) [12].

- La cire de citron (nom INCl: Citrus Medica Limonum (Lemon) Peel Extract) dérive des cires contenues dans l'écorce du fruit. Elle contient des bioflavonoïdes (polyphénols) et porte ainsi des revendications cosmétiques multiples telles que anti-inflammatoire, hydratante, émolliente, anti-cellulite, etc. [13].

\section{Les substituts}

En parallèle de la tendance «produits naturels », plusieurs excipients utilisés depuis longtemps en formulation cosmétique ont vu leur réputation mise à mal. C'est le cas de la vaseline, produit dérivé du pétrole, et de la lanoline et ses dérivés (origine animale).

Le toucher reste la propriété première d'un émollient, et dans le cas de la vaseline, c'est ce paramètre qui a été ciblé pour la mise au point des substituts d'origine végétale [14].

Pour la lanoline et ses dérivés, qui sont largement utilisés dans les applications maquillages, les propriétés apportées sont à la fois cosmétiques (brillant, pouvoir humectant) et techniques (aide à la dispersion de pigment, augmentation de la plasticité des cires) [15].

Les nouvelles alternatives « vertes » proposées aujourd'hui proviennent de la chimie des esters de polyglycérols, qui combinent plusieurs des propriétés de la lanoline, tout en ayant une meilleure stabilité thermique, et présentant des propriétés organoleptiques neutres [16].

\section{Les nouveaux usages}

La bougie de massage est une très bonne illustration de la maîtrise d'un savoir-faire et de son adaptation aux attentes du marché croissant du bien-être.

Constituée d'un mélange d'huiles et de cires d'origine naturelle, cette nouvelle forme galénique présente deux propriétés principales : un point de goutte faible du mélange de corps gras, pour qu'une fois la mèche allumée, la bougie fonde à une température proche de celle de la peau ; associé à une faible pénétration de l'huile de massage obtenue après fusion de la bougie.

\section{Conclusion}

Ces différents exemples de nouvelles matières premières répondent à la fois à la demande du consommateur final, mais aussi aux préoccupations des industriels par rapport à l'évolution des réglementations, et principalement la nouvelle réglementation européenne CE 1907/2006 (REACH) [17], laquelle favorise le développement durable au travers du respect de la santé humaine et de l'environnement. Les perspectives de développement s'inscrivent ainsi dans une logique partagée par toute la chaîne d'approvisionnement, où la traçabilité et l'innocuité d'une matière première (de sa source à sa formulation) deviennent aussi importantes que ses propriétés intrinsèques.

\section{RÉFÉRENCES}

1. ECOCERT. http ://www.ecocert.fr/-Nos-serviceset-notre-savoir-faire.html.

2. QUALITÉ FRANCE. http ://www.qualitefrance.com. 
3. RAMIREZ J, MORONI L. Formulating with exotic butters. Pers Care Mag March 2008 : 13-5.

4. MOULOUNGUI Z, ALFOS C, ROSSIGNOLCASTERA A. Utilisation des lipides non polaires dans les rouges à lèvres : état de l'art et perspectives. OCL 2006 ; 13(5) : 326-8.

5. DE CLERMONT-GALLERANDE H. Evolution des corps gras dans la formulation des rouges à lèvres au cours des quinze dernières années. OCL 2006 ; 13(5) : 322-5.

6. OILS BY NATURE. Fiche Technique Kokum Butter. http ://www.oilsbynature.com/products/ kokum-butter-refined.htm.
7. OILS BY NATURE. Fiche Technique Mowrah Butter. http ://www.oilsbynature.com/products/ mowrah-butter-refined.htm.

8. OILS BY NATURE. Fiche Technique Sal Butter. http ://www.oilsbynature.com/products/salbutter-refined.htm.

9. STRAHL \& PITSCH INC. Fiche Technique Butters. http ://www.spwax.com/newprods.htm.

10. OILS BY NATURE. Fiche Technique Avocado Butter. http ://www.oilsbynature.com/products/ avocado-butter.htm.

11. KAHL. Fiche Technique Mimosa Wax 6602. http ://www.kahlwax.de.

12. KOSTER KEUNEN. Fiche Technique Orange Peel Wax. http ://www.kosterkeunen.com.
13. KOSTER KEUNEN. Fiche Technique Lemon Peel Wax. http ://www.kosterkeunen.com.

14. STRAHL \& PITSCH INC. Fiche Technique « NaturalwaxJellies.http ://www.spwax.com/n ewprods.htm.

15. BARONE S, COHEN I, SCHLOSSMAN M. Lipstick technology. SCC 2006 - Monograph Number 8.

16. ROSSOW COSMÉTIQUES. Fiche Technique Lanoline substitutes (Rossow Cosmétiques pour Sakamoto).

17. COMMISSIONEUROPEENNE. http ://ec.europa.eu/ environment/chemicals/reach/reach_intro.htm. 